\title{
Efecto de la intervención con metformina durante el em- barazo en la diabetes mellitus gestacional en mujeres con síndrome de ovario poliquístico: una revisión sistemática y metanálisis (1)
}

\author{
Zhuo Z, Wang A, Yu H. Effect of metformin intervention during pregnancy on the gestatio- \\ nal diabetes mellitus in women with polycystic ovary syndrome: a systematic review and \\ meta-analysis. J Diabetes Res. 2014; 2014: 381231. Epub 2014 May 21. \\ Análisis Crítico: Sebastián Viguera Torrealba ${ }^{1}$, Jorge A. Carvajal, PhD. ${ }^{1}$ \\ 1 Unidad de Medicina Materno-Fetal, División de Obstetricia y Ginecología. Facultad de Medicina. Pontificia Universidad \\ Católica de Chile.
}

\section{RESUMEN (1)}

La metformina es un sensibilizador a insulina y es efectiva en el tratamiento de la diabetes mellitus tipo 2. Sin embargo, no se ha evaluado las consecuencias funcionales de la administración de metformina durante el embarazo en la diabetes mellitus gestacional (DMG) en mujeres con síndrome de ovario poliquístico (SOP). Para eso efectuamos una revisión sistemática y un metanálisis para determinar el efecto de la metformina sobre la DMG en el SOP. El meta-análisis se realizó en los estudios publicados antes de diciembre de 2013. El meta-análisis examinó si la metformina puede reducir la ocurrencia de DMG en pacientes con SOP usando un modelo de efectos fijos. La razón de chances (Odds Ratio, OR) y el intervalo de confianza del 95\% (IC95\%) se calculó para estimar la fuerza de la asociación. Un total de 13 estudios que incluyeron 5 investigaciones clínicas aleatorizadas (ICA) y 8 no aleatorizados fueron analizados. En última instancia, el análisis de eficacia demostró ausencia de efecto significativo de la metformina, comparado con placebo, sobre la DMG en mujeres con SOP $(\mathrm{OR}=1,07$; IC95\%: 0,60 a 1,92) en las ICA y reducción significativa de la DMG en el grupo metformina en los estudios no aleatorizados (OR=0,19; IC $95 \%$ : $0,13$ a 0,27$)$. En resumen, de acuerdo con los re- sultados de nuestro metanálisis, estrictamente, la metformina no afectó significativamente la DMG en mujeres con SOP, aunque más ICA multicéntricas serían todavía necesarias.

\section{A. Relevancia clínica de la investigación}

El síndrome de ovario poliquístico (SOP) es uno de las patologías endocrinas más frecuentes en la mujeres en edad fértil. Este síndrome se caracteriza por oligoamenorrea, hiperandrogenismo e imagen ecográfica clásica de múltiples quistes ováricos. A lo anterior se suma insulino-resistencia en aproximadamente dos tercios de las pacientes (2). Además, las pacientes con SOP tienen mayor incidencia de patología obstétrica, tales como aborto, parto prematuro, preeclampsia y diabetes gestacional. Un reciente metanálisis publicado el año 2013, concluyó que la incidencia de diabetes gestacional es significativamente mayor en pacientes con SOP (OR=3,43; IC95\%: 2,49 a 4,74) (3).

La metformina, un insulino sensibilizador, ha sido ampliamente usado en el manejo de insulinoresistencia, diabetes mellitus tipo 2 y más recientemente en diabetes mellitus gestacional (DMG). Existe evidencia que el uso de metformina en pacientes embarazadas con DMG, tendría menos resultados obstétricos adversos, comparado con el 
manejo insulínico en estas mismas pacientes (4). Por lo anterior, se ha postulado que por su posible asociación fisiológica, la metformina podría ser una adecuada línea de prevención y tratamiento de DMG en pacientes embarazadas con antecedente de SOP. Lo anterior es de gran relevancia considerando la gran cantidad de complicaciones obstetricias derivadas de la DMG. Los ensayos clínicos aleatorizados no han demostrado el beneficio de la metformina en reducir la incidencia de DMG en mujeres con SOP, sin embargo los estudios observacionales sugieren un posible efecto beneficioso de la metformina en este grupo de pacientes. El presente metanálisis, es la primera revisión sobre este tema que incluye ensayos clínicos aleatorizados (ICA) y estudios observacionales con un metanálisis independiente para cada uno.

\section{ANÁLISIS DE LA INVESTIGACIÓN}

\section{B. El estudio (1)}

Diseño: Revisión sistemática y metanálisis de 13 trabajos publicados entre los años 2002 y 2012 , incluyendo 5 ensayos clínicos aleatorizados. La revisión define en su introducción la pregunta a responder, la cual desglosaremos para su análisis. Pacientes: mujeres embarazadas con diagnóstico pregestacional de SOP. Intervención: tratamiento con metformina como insulino sensibilizador para el manejo de SOP. Comparación: mujeres embarazadas con diagnóstico pregestacional de SOP que no recibieron metformina o que hayan sido manejadas con placebo. Resultado principal a estudiar: ocurrencia de diabetes gestacional. Resultado: el metanálisis de los estudios aleatorizados (ICA) no encontró diferencia estadísticamente significativa para el resultado principal. El metanálisis de los trabajos observacionales demuestra una reducción, estadísticamente significativa, en la incidencia de DMG en mujeres con SOP que usaron metformina, comparado con placebo o no tratamiento (OR=0,19; IC95\%: 0,13 a 0,27).

\section{Análisis crítico}

Validez interna: La búsqueda de información se realizó a partir de 3 fuentes de información diferentes MEDLINE, EMBASE y "Cochrane Central Register of Controlled Trials" La terminología de búsqueda fue adecuada: "pregnancy, pregnant complication, GDM, gestational diabetes mellitus, diabetes, metformin, biguanide, insulin-sensitizing drugs, insulinsensitizers, polycystic ovary syndrome y PCOS". El rango de búsqueda fue amplio, entre 1966 y diciem- bre 2013, sin restricciones de lenguaje. La búsqueda se amplió a partir de la lectura de las secciones de referencia de todos los estudios pertinentes en todos los idiomas, una búsqueda manual de las revistas clave y los resúmenes de las principales reuniones anuales en las áreas de endocrinología y ginecología y obstetricia. Los artículos fueron excluidos del análisis si no tenían: grupos de control adecuados pareados por enfermedad ("disease match control groups") o datos sobre los resultados del embarazo con respecto a diabetes gestacional y la exposición a metformina durante el embarazo o por lo menos en el primer trimestre. Los grupos de control consistieron en mujeres con SOP que no fueron tratados con metformina o placebo. Se realizó una adecuada búsqueda de la literatura disponible con el objetivo de responder la pregunta. Se describió claramente la metodología de búsqueda. Los criterios de inclusión y exclusión de los estudios fueron claros y apropiados. Se explicitan de manera incompleta los sesgos por analizar en los estudios aleatorizados, aunque si se consideraron los puntos mas importante como la aleatorización, el ciego y el análisis por intención de tratar. Sin embargo, no se concluye la evaluación general de sesgo para cada ICA según las recomendaciones de las guías Cochrane.

En cuanto al cálculo de heterogeneidad para evaluar que tan agrupables son los estudios, los autores cometen un error en la interpretación de los resultados. Sabemos que el cálculo de heterogeneidad usando el " $Q$ test - Revman" de Cochrane, concluye a modo general, que los estudios son heterogéneos si se obtiene un $p<0,05$ o 0,1 dependiendo del diseño. En este metanálisis todos los forest plot analizados informan $p>0,05$, es decir que son homogéneos; sin embargo, los autores concluyen que los estudios son heterogéneos, un claro error de interpretación. Aún más, todos los índices de inconsistencia $\left(\mathrm{l}^{2}\right)$ son cercanos a cero, lo que nuevamente nos habla de altos niveles de homogeneidad. En cuanto a la obtención de resultados y su análisis, esta fue informada de forma clara y coherente con el diseño de la revisión y metanálisis. En cuanto a las ICA, en la metodología se explica que de los cinco elegidos, tres son un duplicado por lo que se elige solamente uno de ellos; de los dos que quedan uno es un epianálisis del otro por lo que también se elimina. Finalmente el análisis se realiza a partir de dos ICA. Esto sucedió ya que las cinco ICA son derivados del mismo grupo de investigación, por lo que muchos puntos eran compartidos, incluyendo diseño, pacientes y resultados. De las dos ICA finalmente analizados, el resultado informa que no hay diferencia estadísticamente sig- 
nificativa del uso de metformina; más aún, el OR del metanálisis es prácticamente uno.

Por el contrario, considerando los ocho estudios observacionales, el resultado del metanálisis otorga una diferencia estadísticamente significativa a beneficio del uso de metformina (OR=0,19; IC95\%: $0,13$ a 0,27$)$. Sabemos que los estudios observacionales aportan evidencia de menor calidad que los aleatorizados, sin embargo, las ICA incluidas en esta revisión son solo dos, y uno de ellos con bajo número de mujeres incluidas.

Validez externa: Los resultados si podrían ser extrapolables a nuestra población. A pesar que la población de los estudios analizados es nórdica escandinava, diferente de nuestra población que se compone principalmente de un mestizaje españolindígena, las definiciones de SOP utilizadas son las estándar, lo que las hace poblaciones sean similares. En cuanto a la intervención, la metformina es un medicamento localmente utilizado, aceptado por Instituto de Salud Pública y ampliamente disponible. En el estudio no explicitan la definición de diabetes gestacional, pero no debería influir en la reproducibilidad local, ya que las definiciones de esta patología son similares a nivel internacional.

Conclusiones: Esta revisión y metanálisis, tiene una pregunta estructurada, claramente anunciada y completa. La búsqueda fue adecuada y la me- todología correcta. Sin embargo se explicitan de manera incompleta los sesgos por analizar, especialmente en los randomizados. A pesar que la conclusión obtenida nos parece correcta, en la discusión se cometen errores de interpretación con respecto a algunos resultados. El presente trabajo no presenta evidencia suficiente para cambiar la práctica clínica, por ello, no recomendamos el uso sistemático de metformina en paciente embarazadas con antecedente de síndrome de ovario poliquístico.

\section{REFERENCIAS}

1. Zhuo Z, Wang A, Yu H. Effect of metformin intervention during pregnancy on the gestational diabetes mellitus in women with polycystic ovary syndrome: a systematic review and meta-analysis. J Diabetes Res 2014; 2014: 381231.

2. DeUgarte CM, Bartolucci AA, Azziz R. Prevalence of insulin resistance in the polycystic ovary syndrome using the homeostasis model assessment. Fertil Steril 2005;83(5):1454.

3. Qin JZ, Pang LH, Li MJ, Fan XJ, Huang RD, Chen HY. Obstetric complications in women with polycystic ovary syndrome: a systematic review and meta-analysis. Reprod Biol Endocrinol. 2013;11:56.

4. Goh JE, Sadler L, Rowan J. Metformin for gestational diabetes in routine clinical practice. Diabet Med. 2011 Sep;28(9):1082-7. 